\title{
INFORMATION, STABILIZATION, AND WELFARE: THE CASE OF SUNSPOTS*
}

\author{
Subir Chattopadhyay**
}

WP-AD 99-02

Correspondence: Departamento de Fundamentos del Análisis Económico

Universidad de Alicante, 03071 Alicante, SPAIN

Editor: Instituto Valenciano de Investigaciones Económicas, s.a.

Primera Edición Febrero 1999

ISBN: 84-482-2021-8

Depósito Legal: V-770-1990

IVIE working-papers offer in advance the results of economic research under way in order to encourage a discussion process before sending them to scientific journals for their final publication.

${ }^{*}$ This paper is based on my dissertation at SUNY Stony Brook. I would like to thank my supervisor, Professor T. J. Muench, for his advice, comments, and encouragement. I am grateful to S. Chatterji whose comments did much to improve the exposition. I thank an anonymous referee for suggesting an interesting and nontrivial extension. Earlier versions of the paper were presented in seminars at Alicante, Barcelona (Autonoma), CORE, ISI Delhi, Jerusalem, Madrid (Carlos III), Paris, and Tel Aviv. Financial support in the form of a Lady Davis Fellowship at the Hebrew University, and additional support from the IVIE, DGICyT via grant number PB94-1504, and the European Commission via grant number TMR/FMRX-CT95/0055, is gratefully acknowledged.

** University of Alicante 


\title{
INFORMATION, STABILIZATION, AND WELFARE: \\ THE CASE OF SUNSPOTS
}

\section{Subir Chattopadhyay}

WP-AD 99-02

\begin{abstract}
The stationary sunspot equilibria of a simple OLG economy with heterogeneous agents are considered. These equilibria are known to be suboptimal. The focus of the paper is on the efficacy, based on welfare economic considerations and informational requirements, of government "policy" in such an environment.

The main result is that knowledge of (a) the sunspot equilibrium net trades, (b) weak interval type information on two parameters, and (c) weak set type information on the location of some optimal stationary allocation, is sufficient to induce a competitive equilibrium which is a Pareto optimal Pareto improvement over the sunspot allocation which has the further property of reaching a Pareto optimal stationary allocation in finite time.

The results are interpreted as demonstrating that in a simple model with a sunspot environment, policy is very effective and that welfare economic considerations lead to "stabilization."
\end{abstract}

JEL Nos.: D52, D61, D84, D91, E61, E63

KEYWORDS: Stationary Sunspots, Overlapping Generations, Pareto Optimal Improvements, Golden Rule, Stabilization 


\section{Introduction}

This paper evaluates the potential for policy intervention in a simple overlapping generations economy undergoing sunspot fluctuations. We show that interventionist policy is effective in the strong sense that it can lead to Pareto improving, Pareto optimal allocations which can be induced using relatively weak information.

\subsection{The model and the results}

The class of economies considered are multi-agent, stationary, pure exchange, overlapping generations (henceforth, OLG) economies in which one good is traded in each period. Money is used to transfer income across periods. Agents maximize expected utility and are risk averse. The only other assumption made is that consumption when old is a normal good.

There is a government in the economy which does not produce or consume any goods; however, the government can level real lump-sum taxes and controls the quantity of money in the economy by making lump-sum transfers and interest payments. So, it is in a position to affect the allocations that agents receive by appropriate choice of its fiscal-monetary policy. We assume that the government does not have costless access to information about agents' preferences and endowments.

We are concerned with the stationary sunspot equilibria (henceforth, SSE) of this model. A sunspot equilibrium, following Cass and Shell [2], is a rational expectations equilibrium in which uncertainty matters only because agents believe that it does even though preferences and endowments are deterministic. It is well known that SSE exist in the class of economies considered in this paper (see, e.g. Azariadis [1]); furthermore, under a standard criterion of efficiency, they are suboptimal (see, e.g. Peck [13]).

The policy problem treated in this paper can now be described. We postulate that the economy is already in an SSE with two states and, given the suboptimality of this equilibrium, the objective is to give an axiomatic characterization of the kind of information necessary in order to induce an allocation which is Pareto optimal and Pareto improving over the SSE allocation.

As a preliminary to our main results, we prove the existence of two parameters which are defined for each economy and each SSE allocation. In terms of interpretation, the first parameter gives an upper bound on the maximum amount of either commodity that agents of every type of every generation would be willing to give up in order to avoid the sunspot lottery, i.e. something like a uniform risk premium. The second parameter is used to define a lower bound on the period beyond which all generations would prefer, in comparison to the sunspot lottery, the consumption vector corresponding to a particular stationary allocation which is near the consumption vector corresponding to an optimal stationary allocation. Each of these parameters defines one boundary of an interval where the other boundary is known a priori. We say that we have "interval type information" if we know of some point in the interval specified by each of these two parameters. 
Our Theorem says that, (a) the information that is contained in the time series of the SSE net trades, together with (b) interval type information, and (c) approximate knowledge about the location of the net trade associated with an optimal stationary allocation, is sufficient to compute a Pareto improvement which has the property of giving all but a finite number of agents a stationary consumption vector (the target). Now allowing agents to trade in competitive loan markets only (so we look at the nonmonetary equilibria), with the previously computed Pareto improvement as the endowment stream, necessarily gives a Pareto optimal allocation. In this way we acheive the goal of inducing a Pareto optimal allocation which also Pareto improves over the initial SSE. In the special case where there is only one type of agent and the exact location of the Golden Rule is known, the Golden Rule consumption vector serves as the target. ${ }^{1}$

\subsection{Discussion of the results and the model}

The results of this paper show that, given the structure of the economy considered, interventionist policy can be effective, and that "stabilization" could have foundations in welfare economics. We base our conclusion on our belief that policies should be evaluated on the basis of (i) the existence of allocations which are both Pareto optimal and Pareto improving, and (ii) an axiomatic characterization of the information required in order to implement these allocations. But in order to evaluate the work, we need to discuss alternative specifications of information which might deliver the result; we also need to discuss the way in which we have modeled the government.

In our framework, the informational requirements for computing only Pareto improvements, or only Pareto optimal allocations, are fairly weak; the problem gets interesting when one wants both. Notice that improvements typically require local information (or a global property of preferences like risk aversion used locally) like local recoverability of preferences from demand functions, while optimality is a global notion. ${ }^{2}$ The informational requirements in the Theorem provide the right mix of local and global information; whether they are weak or otherwise is subjective but it is obvious that some preference information must be given to the government since it uses a Pareto criterion, and easy counter-examples can be given when one or the other requirement of the Theorem is dropped.

Alternatively, one could ask whether welfare improvements can be induced based on only aggregate information regarding certain statistics of the distribution of agents'

\footnotetext{
${ }^{1}$ As we indicate in Remark 5, for a "generic" economy, all but a finite number of agents of each type receive a consumption vector which is one of a finite collection of vectors (none of which need be the same as the target); as a special case, beyond a certain point in time, each agent of a given type receives the same vector.

${ }^{2}$ For example, Geanakoplos and Polemarchakis [7] look at a two period model with incomplete markets, show that the resulting allocation is generically constrained suboptimal, and ask how much information a planner will need before she can effect an improvement (not an optimal improvement). Their solution, Geanakoplos and Polemarchakis [8], is based on conditions guaranteeing the local recoverability of preferences from demand functions and is quite different from the approach taken in this paper.
} 
characteristics. Unfortunately, when one uses the Pareto criterion, almost by definition one is forced to ask for some individual specific information; this usually means that, at the very least, we need to know the agents net trades and usually a little more. In this paper we have heterogeneous households but the only information which we need which is specific to the households is (i) information about their net trades and (ii) information about the location of an optimal stationary net trade for their type; the rest of the information that we use is uniform in nature in the sense that it can be obtained once one knows the broad outlines of the agents' characteristics, hence our claim that the informational requirements of the result we obtain are relatively mild given the objective that we start with.

We find that "stabilization" is a result of our objective which was to find easily induced Pareto optimal improvements. This is an insight which appears to be robust to model specification since, given the incomplete information environment that we are considering, and the fact that the First Welfare Theorem fails in OLG economies, it is natural to use a stationary allocation as a target to be reached in finite time; this is about the only robust way of guaranteeing optimality of the entire allocation by converting the economy into one where the infinite horizon does not matter.

Turning to the model, one could say that in such a simple environment, the government will not suffer from incompleteness of information since it will be composed of the agents who populate the economy. A first answer to this criticism is that the model and results can be extended (see Section 1.3); but a more reasonable answer is that those who design macroeconomic policies clearly suffer from the type of lack of information that has been modeled in this paper and to gauge whether they can be successful in designing desirable policies in realistic environments, one should start with simple, possibly unrealistic, specifications which is what we do in this paper.

Overall, we view the paper as exploratory and hope that it will stimulate interest in a systematic approach to the problem of the role of policy intervention in actual economies. One would guess that analysis of more general models will lead to the conclusion that the goals of interventionist policy should be less lofty, not because no-intervention is the best of all worlds, but because the potency of policy is severely limited by informational considerations. $^{3}$

\subsection{Extensions and drawbacks}

Our analysis applies to all SSE, "local" or otherwise, and also to the case of SSE with more than two states, under a standard mixing condition on the Markov process. Also, under mild additional conditions, it is possible to define the two parameters that we use uniformly across different equilibria and different economies.

An extension to multi-good economies is desirable. ${ }^{4}$ The problem is more difficult

\footnotetext{
${ }^{3}$ Geanakoplos and Polemarchakis [7] also stress this point.

${ }^{4}$ However, note that the one good case is often regarded as the one relevant for macroeconomics.
} 
relative to the one good case since the conditions for optimality are much more stringent. However, it might be possible to replicate the strategy that we use in the one good case of computing improvements which reach a stationary target in finite time and then allow markets to operate, with the new endowments, in order to obtain optimality. Clearly, such allocations exist; the question of interest is whether there are acceptable informational requirements which will let us compute the initial improving allocation.

It is also of interest to identify conditions under which one can reach the target stationary allocation in an optimal and improving manner without explicit knowledge about its location. Such a result can be obtained by using the monetary policy rule proposed by Grandmont [10]. ${ }^{5}$

There are two problems with our approach that we would like to mention. We do not ask how the government could possibly know that the economy is caught in an SSE; nor do we ask how the government learns about the structure of the economy which we formalize as assumptions.

\subsection{Related literature}

A problem which is related to the problem treated in this paper is that of "stabilizing sunspots" (e.g. Grandmont [10] and [11], and Woodford [14]). The objective there is to neutralize sunspots; consequently, welfare issues are typically not considered. The result is usually in the form of a policy rule which often has the property that if the rule is announced before economic activity begins, and the initial conditions are suitable, then interference by the government is never actually necessary. However, if the economy is already undergoing sunspot fluctuations then interference would be necessary and the resulting allocations need be neither improving nor optimal. Furthermore, the informational requirements of the policies proposed are often quite stringent.

The paper is organized as follows: Section 2 introduces the economy, its perfect foresight equilibria, and the existence and welfare properties of SSE. Section 3.1 introduces two parameters which are used in our construction of optimal improvements. Section 3.2 presents the main result and its proof. Other proofs are collected in Section 4.

\section{The economy}

\subsection{The model $^{6}$}

Consider a simple OLG economy. Time is discrete, $t=0,1,2, \cdots$, and there is one good in each period. At each $t \geq 1, H$ "types" of agents are born and live for that and the next period. Types are assumed to be identical across generations. Denote the consumption vector of agent $h$ of generation $t$ by $\left(c_{h ; 1, t}, c_{h ; 2, t}\right) \in R_{+}^{2}$ and call it an outcome. The preferences of every type will be assumed to be representable by a von

\footnotetext{
${ }^{5}$ See Chattopadhyay [4] where such a result is obtained in the classical OLG framework with one type of household.

${ }^{6}$ For details and references on the OLG model see, e.g. Geanakoplos [6] and Geanakoplos and Polemarchakis [9].
} 
Neumann Morgenstern utility function $U^{h}\left[c_{h ; 1, t}, c_{h ; 2, t}\right]$ defined on $R_{+}^{2}$. Endowments will be denoted by $\left(\omega_{h ; 1}, \omega_{h ; 2}\right)$.

Assumption A.1: For every $h \in H, U^{h}: R_{+}^{2} \rightarrow R$ is $C^{2}$ with $D U^{h}>>0$ and $D^{2} U^{h}$ negative definite, on $R_{++}^{2}$; the closure of every indifference curve which passes through $R_{++}^{2}$ is contained in $R_{++}^{2}$.

Assumption A.2: For all $h \in H,\left(\omega_{h ; 1}, \omega_{h ; 2}\right) \in R_{++}^{2}$.

Assumption A.3: (Second period consumption is a normal good)

$U_{1}^{h}\left[c_{h ; 1}, c_{h ; 2}\right] U_{12}^{h}\left[c_{h ; 1}, c_{h ; 2}\right]>U_{2}^{h}\left[c_{h ; 1}, c_{h ; 2}\right] U_{11}^{h}\left[c_{h ; 1}, c_{h ; 2}\right], \quad \forall\left(c_{h ; 1}, c_{h ; 2}\right) \in R_{++}^{2}$.

A.1 and A.2 are standard technical requirements and will be treated as maintained hypotheses. A.3 is necessary in order to let us prove our results. ${ }^{7}$

Also, let the agents born at $t=0$ have a monotone increasing utility function $u^{h}\left(c_{h ; 2,0}\right)$ and second period endowment $\omega_{h ; 2}$.

Further assume that an agent of type $h$ born at date $t$ receives a lump-sum transfer (possibly negative) of fiat money from the government when old, $\tau_{h ; t}$. A monetary policy will be given by a collection of sequences of transfers. If $\sum_{h \in H} \tau_{h ; 0}=M \neq 0, \tau_{h ; t}=0$, for all $h \in H$ and for all $t=1,2,3, \cdots$, then we have a constant money supply.

\subsection{Golden Rules}

We say that $\left(\left(\omega^{h}\right)_{h \in H}\right) \in R_{+}^{H}$ is a distribution of the endowments if $\sum_{h \in H} \omega^{h}=\sum_{h \in H} \omega_{h ; 1}+\sum_{h \in H} \omega_{h ; 2}$.

Given such an $\left(\left(\omega^{h}\right)_{h \in H}\right) \in R_{+}^{H}$, let $\left(\left(c_{h ; 1}^{G R}\left(\omega^{h}\right), c_{h ; 2}^{G R}\left(\omega^{h}\right)\right)_{h \in H}\right) \in R_{+}^{2 H}$, denote the Golden Rule allocation, the allocation that solves the problem

$\max U^{h}\left[c_{h ; 1}, c_{h ; 2}\right] \quad$ subject to $\quad\left(c_{h ; 1}, c_{h ; 2}\right) \in R_{+}^{2}$ and $c_{h ; 1}+c_{h ; 2}=\omega^{h}$.

So the Golden Rule gives maximal utility to the different agent types in the class of stationary allocations which respect the given distribution of the total endowment among the different types; the MRS at the Golden Rule is one.

\subsection{Perfect foresight}

Under perfect foresight, all the future prices and transfers are known with certainty. A sequence of prices such that agents optimize and markets clear at each date will be called a perfect foresight equilibrium (PFE) sequence:

$$
\begin{array}{r}
\sum_{h \in H} c_{h ; 1, t+1}\left(p_{t+1}, p_{t+2} ; \tau_{h ; t+1}\right)+\sum_{h \in H} c_{h ; 2, t}\left(p_{t}, p_{t+1} ; \tau_{h ; t}\right)= \\
=\sum_{h \in H} \omega_{h ; 1}+\sum_{h \in H} \omega_{h ; 2} \text { for all } t=0,1,2,3, \cdots .
\end{array}
$$

Remark 1: Under Assumptions 1 and 2, PFE exist. Also, the Second Welfare Theorem holds for the class of economies considered; in particular, any Golden Rule allocation can be supported as a monetary steady state provided that the initial distribution of money holdings (different components of which could be positive or negative depending on the allocation which we choose to support) is appropriately chosen.

\footnotetext{
${ }^{7}$ If the utility function is assumed to be additively separable then A.1 implies A.3.
} 


\subsection{Stationary sunspot equilibria ${ }^{8}$}

\subsubsection{Existence}

Consider a time-homogeneous Markov process with two states, $s \in\{\alpha, \beta\}$.

$$
\Pi=\left(\begin{array}{cc}
\pi^{\alpha \alpha} & 1-\pi^{\alpha \alpha} \\
1-\pi^{\beta \beta} & \pi^{\beta \beta}
\end{array}\right)
$$

is the transition matrix where $\pi^{s s^{\prime}}$ is the probability of being in state $s^{\prime}$ in the next period conditional on being in state $s$ in the current period. The process describes the evolution of an "extrinsic" state, i.e. preferences and endowments continue to be deterministic. But agents believe that the random variable affects prices. We focus on equilibria in which prices depend on only the current realization of the extrinsic state. An SSE is a pair of prices and associated allocations such that agents' beliefs about prices are fulfilled and their optimizing demands clear markets.

Given today's state, $s \in\{\alpha, \beta\}$, the agents act so as to ${ }^{9}$

$$
\begin{aligned}
& \max _{c_{h ; 1}^{s}, c_{h ; 2}^{s \alpha}, c_{h ; 2}^{s \beta}, m_{h}^{s}} \pi^{s \alpha} U^{h}\left[c_{h ; 1}^{s}, c_{h ; 2}^{s \alpha}\right]+\pi^{s \beta} U^{h}\left[c_{h ; 1}^{s}, c_{h ; 2}^{s \beta}\right], \quad \text { subject to } \\
& p^{s} c_{h ; 1}^{s}+m_{h}^{s}=p^{s} \omega_{h ; 1}, \quad p^{\alpha} c_{h ; 2}^{s \alpha}=p^{\alpha} \omega_{h ; 2}+m_{h}^{s}, \quad p^{\beta} c_{h ; 2}^{s \beta}=p^{\beta} \omega_{h ; 2}+m_{h}^{s} .
\end{aligned}
$$

Goods market clearing requires that, for $s \in\{\alpha, \beta\}$,

$$
\sum_{h \in H} c_{h ; 1}^{s}+\sum_{h \in H} c_{h ; 2}^{\alpha s}=\sum_{h \in H} \omega_{h ; 2}+\sum_{h \in H} \omega_{h ; 2}=\sum_{h \in H} c_{h ; 1}^{s}+\sum_{h \in H} c_{h ; 2}^{\beta s},
$$

with the the implication that $\sum_{h \in H} c_{h ; 2}^{\alpha s}=\sum_{h \in H} c_{h ; 2}^{\beta s}:=c_{2}^{s}, s \in\{\alpha, \beta\}$.

Definition 1: Given preferences, endowments, and a constant money supply $M \neq 0$, the tuple $\left(p^{\alpha}, p^{\beta} ;\left(c_{h ; 1}^{\alpha}, c_{h ; 1}^{\beta}, c_{h ; 2}^{\alpha \alpha}, c_{h ; 2}^{\alpha \beta} c_{h ; 2}^{\beta \alpha}, c_{h ; 2}^{\beta \beta}\right)_{h \in H}\right)$ will constitute an SSE for $\Pi$ if:

(i) the demands expressed by the agents at those prices clear the markets;

(ii) $p^{\alpha} \neq p^{\beta} \neq 0$ and $m_{h}^{\alpha} \neq m_{h}^{\beta}$ for all $h \in H$;

(iii) $\Pi>>\left(\begin{array}{ll}0 & 0 \\ 0 & 0\end{array}\right)$.

The second condition rules out SSE that are trivial for some agent (it holds "generecially"). The third condition essentially rules out deterministic cycles of period two from being labelled as SSE. Sufficient conditions for the existence of SSE are well known (see, e.g. Azariadis [1] for the case with only one type of agent).

\subsubsection{Welfare properties}

Before we can analyse the welfare properties of SSE we need to specify a criterion of dominance and optimality. We propose to use the weakest criterion which renders all SSE inefficient; this ensures that the problem which we wish to treat is non-vacuous. ${ }^{10}$ The criterion is of the ex-ante sort and dominance is defined by comparing the sequence of conditional expected utility levels, conditional on the state in some initial period.

\footnotetext{
${ }^{8}$ See Chiappori and Guesnerie [5] and Guesnerie and Woodford [12] for surveys of the literature.

${ }^{9}$ Notation used: for stationary stochastic variables, superscripts represent the state.

${ }^{10}$ We follow Chattopadhyay [3] Section 2.3.2.
} 
Without loss of generality the initial period is taken to be period one. Optimality is defined as usual, i.e. the non-existence of dominating allocations. We refer to stochastic allocations in our definitions without defining them formally; it suffices to take them to be contingent on the sunspot process (see Remark 2). ${ }^{11}$

We need some notation first. For $t \geq 2$, let $\Pi_{t}:=\left(\Pi^{\prime}\right)^{t-1}$ be the matrix which gives the probability, $\pi_{t}^{s s^{\prime}}$, of being in state $s^{\prime}$ in period $t$ given that the economy started in state $s$ in period 1, i.e. the conditional probability distribution.

Consider a particular stochastic allocation $\left\{\left(c_{h ; 1, t}, c_{h ; 2, t}\right)_{h \in H}\right\}_{t=1}^{t=+\infty}$. We can define the sequence of conditional ex-ante expected utility levels,

$$
E U\left[\left\{\left(c_{1, t}, c_{2, t}\right)_{h \in H}\right\}_{t=1}^{t=+\infty}\right]:=\left\{\left(E U_{j}^{h}\left[\left\{\left(c_{h ; 1, t}, c_{h ; 2, t}\right)_{h \in H}\right\}_{t=1}^{t=+\infty}\right]\right)_{h \in H}\right\}_{j=1}^{j=+\infty},
$$

from this allocation where $E U_{j}^{h}\left[\left\{\left(c_{h ; 1, t}, c_{h ; 2, t}\right)_{h \in H}\right\}_{t=1}^{t=+\infty}\right]$ is the expected utility of the agent of type $h$ born in period $j$ where the expectation is taken relative to the conditional distribution (conditional on information available at $t=1$ ) generated by the process on which the allocation under consideration is contingent.

Definition 2: A feasible allocation is a sequence of (possibly random) vectors in $R_{+}^{2 H}$, $\left\{\left(c_{h ; 1, t}, c_{h ; 2, t}\right)_{h \in H}\right\}_{t=1}^{t=+\infty}$, and $\left(c_{h ; 2,0}\right)_{h \in H} \in R_{+}^{H}$, such that $\sum_{h \in H} c_{h ; 1, t}+\sum_{h \in H} c_{h ; 2, t-1}=$ $\sum_{h \in H} \omega_{h ; 1}+\sum_{h \in H} \omega_{h ; 2} \quad$ for each realization and $\forall t=1,2,3, \cdots$.

Definition 3: A feasible allocation $\left\{\left(c_{h ; 1, t}, c_{h ; 2, t}\right)_{h \in H}\right\}_{t=1}^{t=+\infty}$ and $\left(c_{h ; 2,0}\right)_{h \in H}$ dominates the feasible allocation $\left\{\left(\tilde{c}_{h ; 1, t}, \tilde{c}_{h ; 2, t}\right)_{h \in H}\right\}_{t=1}^{t=+\infty}$ and $\left(\tilde{c}_{h ; 2,0}\right)_{h \in H}$, if $u^{h}\left(c_{h ; 2,0}\right) \geq u^{h}\left(\tilde{c}_{h ; 2,0}\right)$ for all $h \in H$ and $E U_{j}^{h}\left[\left\{\left(c_{h ; 1, t}, c_{h ; 2, t}\right)_{h \in H}\right\}_{t=1}^{t=+\infty}\right] \geq E U_{j}^{h}\left[\left\{\left(\tilde{c}_{h ; 1, t}, \tilde{c}_{h ; 2, t}\right)_{h \in H}\right\}_{t=1}^{t=+\infty}\right]$ for all $h \in H$ and for all $j=1,2,3, \cdots$ with strict inequality for at least one agent type $h$ at some $j$ or 0 .

Definition 4: A feasible allocation, $\left\{\left(c_{h ; 1, t}, c_{h ; 2, t}\right)_{h \in H}\right\}_{t=1}^{t=+\infty}$ and $\left(c_{h ; 2,0}\right)_{h \in H}$, is Pareto optimal if there is no other feasible allocation, $\left\{\left(\tilde{c}_{h ; 1, t}, \tilde{c}_{h ; 2, t}\right)_{h \in H}\right\}_{t=1}^{t=+\infty}$ and $\left(\tilde{c}_{h ; 2,0}\right)_{h \in H}$, which dominates it.

The stochastic sequence corresponding to the SSE allocation will be denoted by $s($.$) ,$ while the sequence of ex-ante expected utility levels from the SSE will be denoted by $E U^{h}[s()$.$] where$

$$
\begin{aligned}
& E U_{t}^{h}[s(.)]=\pi_{t}^{s \alpha} U^{h}(\alpha)+\pi_{t}^{s \beta} U^{h}(\beta) \\
& U^{h}(\alpha):=\pi^{\alpha \alpha} U^{h}\left[c_{h ; 1}^{\alpha}, c_{h ; 2}^{\alpha \alpha}\right]+\pi^{\alpha \beta} U^{h}\left[c_{h ; 1}^{\alpha}, c_{h ; 2}^{\alpha \beta}\right] \\
& U^{h}(\beta):=\pi^{\beta \alpha} U^{h}\left[c_{h ; 1}^{\beta}, c_{h ; 2}^{\beta \alpha}\right]+\pi^{\beta \beta} U^{h}\left[c_{h ; 1}^{\beta}, c_{h ; 2}^{\beta \beta}\right] .
\end{aligned}
$$

Consider a given SSE and define the Expected allocation by

$\bar{c}_{h ; 2,0}:=c_{h ; 2}^{s} ; \quad \bar{c}_{h ; 1, t}:=\pi_{t}^{s \alpha} c_{h ; 1}^{\alpha}+\pi_{t}^{s \beta} c_{h ; 1}^{\beta} ;$

$\bar{c}_{2, t}:=\pi_{t}^{s \alpha}\left(\pi^{\alpha \alpha} c_{h ; 2}^{\alpha \alpha}+\pi^{\alpha \beta} c_{h ; 2}^{\alpha \beta}\right)+\pi_{t}^{s \beta}\left(\pi^{\beta \alpha} c_{h ; 2}^{\beta \alpha}+\pi^{\beta \beta} c_{h ; 2}^{\beta \beta}\right) \quad \forall t=1,2,3, \cdots$,

where $\pi_{1}^{s s^{\prime}}=0$ or 1 as the state in period one is known. Note that the Expected allocation is a function of the state in period one.

\footnotetext{
${ }^{11}$ Deterministic allocations are, as usual, special cases of stochastic allocations.
} 
Following Cass and Shell [2] and Peck [13], we have that the SSE allocation is not Pareto optimal since it is dominated by the Expected allocation. Formally

Proposition 1: If $s($.$) is an SSE allocation then it is not Pareto optimal.$

Remark 2: Clearly, any feasible stochastic allocation can be dominated by the corresponding "certainty" or "expected" allocation. Hence, the search for Pareto optimal allocations can be restricted to the class of certainty allocations.

We define $\pi^{\alpha}, \pi^{\beta}$, and $E^{*} U^{h}[s()$.$] by$

$$
\pi^{\alpha}:=\frac{1-\pi^{\beta \beta}}{2-\pi^{\alpha \alpha}-\pi^{\beta \beta}}, \pi^{\beta}:=\left(1-\pi^{\alpha}\right), \quad E^{*} U^{h}[s(.)]:=\pi^{\alpha} U^{h}(\alpha)+\pi^{\beta} U^{h}(\beta) .
$$

\section{Pareto optimal improvements}

In this section we prove our Theorem. We state a set of informational assumptions that is sufficient to induce a competitive allocation that is Pareto optimal, that is Pareto improving over the SSE, and exhibits a form of stationarity after finite time. In particular, if a certain "target" Golden Rule net trade vector is known then that Golden Rule vector is reached in finite time.

But first, in Section 3.1, we prove some preliminary results and state the informational assumptions made.

\subsection{Preliminaries}

Lemma 4.1 in Section 4 studies the limiting behaviour of the Expected allocation sequence and the sequence of ex-ante expected utilities from the SSE. It shows that the sequence of probabilities defined in Subsection 2.4.2, $\left(\pi_{t}^{s \alpha}, \pi_{t}^{s \beta}\right)$, converges to $\left(\pi^{\alpha}, \pi^{\beta}\right)$, a limit stationary distribution which is independent of the starting state; that due to linearity in the probabilities, the Expected allocation, $\left(\bar{c}_{h ; 1, t}, \bar{c}_{h ; 2, t}\right)$, and the sequence of ex-ante expected utilities from the SSE allocation, $E U_{t}^{h}[s()$.$] , converge to the stationary$ outcome $\left(\bar{c}_{h ; 1}^{*}, \bar{c}_{h ; 2}^{*}\right)$ and to $E^{*} U^{h}[s()$.$] respectively; and that Jensen's Inequality con-$ tinues to hold in the limit. We use these properties to prove the existence of a parameter which will be used in our construction.

Proposition 2: ${ }^{12}$ There exists a finite number $\delta^{*}>0$ such that, uniformly in $t$, $\forall \delta \in\left(0, \delta^{*}\right], \forall h \in H,\left(c_{h ; 1}, c_{h ; 2}\right) \in B_{\delta}\left(\bar{c}_{h ; 1, t}, \bar{c}_{h ; 2, t}\right) \quad \Rightarrow \quad U^{h}\left[c_{h ; 1}, c_{h ; 2}\right] \geq E U_{t}^{h}[s()$.$] .$

The parameter $\delta^{*}$ can be interpreted as the largest amount (in terms of either commodity) of the Expected outcome that agents of every type in every generation will be willing to forego in order to avoid the ex-ante SSE lottery.

In what follows, it will be useful to have some notation to distinguish net trades from consumption vectors (outcomes). So, from now on, $(y, z)$ will denote net trades for some agent type $h$, i.e. $(y, z):=\left(c_{h ; 1}-\omega_{h ; 1}, c_{h ; 2}-\omega_{h ; 2}\right)$.

\footnotetext{
${ }^{12}$ Notation: $B_{r}\left(x_{1}, x_{2}\right)$ denotes the closed ball of radius $r$ with center at $\left(x_{1}, x_{2}\right)$.
} 
We turn to a formal statement of a set of "informational assumptions."

Information I.1 "Market:" $p^{\alpha}, p^{\beta}$, П and $\left(\left(m_{h}^{s}\right)_{s \in\{\alpha, \beta\}, h \in H}\right)$ are known numbers.

Remark 3: I.1 allows the computation of the net trades for the entire Expected allocation, $\left\{\left(\left(\bar{c}_{h ; 1, t}-\omega_{h ; 1}, \bar{c}_{h ; 2, t}-\omega_{h ; 2}\right)_{h \in H}\right)\right\}_{t=1}^{t=+\infty}$ and $\left(\left(\bar{c}_{h ; 2,0}-\omega_{h ; 2}\right)_{h \in H}\right)$, since the SSE net trades can be computed from the state dependent money demands and the SSE prices (using the agents' budget constraints); I.1 also allows the computation of the invariant distribution of the Markov process (using Lemma 4.1). Notice that the transition probabilities can be estimated given data on SSE prices.

Information I.2 "Sufficient statistic:" $\delta \in\left(0, \delta^{*}\right]$, where $\delta^{*}$ satisfies the requirements of Proposition 2, is known.

Before stating the remaining informational assumptions we give an intuitive description of their content. For each type of agent $h \in H$, we will consider two disjoint subsets of the set of net trades where the first of these subsets, to be called $A_{h}^{*}$, gives a utility level which is "almost" the same as $E^{*} U^{h}[s()$.$] , while the second, to be called A_{h}$, contains the Golden Rule net trade corresponding to the distribution of endowments induced by the limiting value of the Expected allocation. Lemma 1 shows that there is a period, to be called $\widehat{N}$, such that for all $h \in H$, the agent of type $h$ born at dates later than $\widehat{N}$ would prefer any outcome in $A_{h}$ to his SSE lottery.

Formally, for each type of agent $h \in H$, define the set $A_{h}^{*}$ to be

$A_{h}^{*}:=\left\{\left(c_{h ; 1}, c_{h ; 2}\right) \in R_{+}^{2} \mid E^{*} U^{h}[s()]-.\epsilon \prime \leq U^{h}\left[c_{h ; 1}, c_{h ; 2}\right] \leq E^{*} U^{h}[s()]+.\epsilon \prime\right\}-\left\{\left(\omega_{h ; 1}, \omega_{h ; 2}\right)\right\}$,

a closed set in $R^{2}$ where $\epsilon$ is sufficiently small so as to ensure that

$$
\left(\left(c_{h ; 1}^{G R}\left(\bar{c}_{h ; 1}^{*}+\bar{c}_{h ; 2}^{*}\right)-\omega_{h ; 1}, c_{h ; 2}^{G R}\left(\bar{c}_{h ; 1}^{*}+\bar{c}_{h ; 2}^{*}\right)-\omega_{h ; 2}\right) \notin A_{h}^{*} .\right.
$$

Information I.3 "Golden Rule:" For each $h \in H$, a set $A_{h} \subset R^{2}$ is known where i) $A_{h}$ is closed and convex, ii) $A_{h} \cap A_{h}^{*}=\emptyset$, and iii) $\left(\left(c_{h ; 1}^{G R}\left(\bar{c}_{h ; 1}^{*}+\bar{c}_{h ; 2}^{*}\right)-\omega_{h ; 1}, c_{h ; 2}^{G R}\left(\bar{c}_{h ; 1}^{*}+\bar{c}_{h ; 2}^{*}\right)-\omega_{h ; 2}\right) \in A_{h}{ }^{13}\right.$

¿From now on let $Q_{h}$ be the compact square

$$
Q_{h}:=\left\{(y, z) \in R^{2} \mid-\omega_{h ; 1} \leq y \leq \sum_{h \in H}\left(\omega_{h ; 1}+\omega_{h ; 2}\right),-\omega_{h ; 2} \leq z \leq \sum_{h \in H}\left(\omega_{h ; 1}+\omega_{h ; 2}\right)\right\}
$$

Let $\left(y^{A_{h}}, z^{A_{h}}\right)$ be a least preferred net trade for the agent of type $h$ in the compact set $A_{h} \cap Q_{h} \cdot{ }^{14}$

\footnotetext{
${ }^{13}$ From Lemma 4.1 (ii) and (iv), for each $h \in H$, there is some stationary allocation, satisfying the endowment distribution condition $c_{h ; 1}+c_{h ; 2}=\bar{c}_{h ; 1}^{*}+\bar{c}_{h ; 2}^{*}$, which is strictly preferred to the limiting SSE lottery. Now the existence of the sets $A_{h}$, for $h \in H$, follows from the definition of the sets $A_{h}^{*}$. Also, given A.1, convexity and continuity of preferences, the requirement that that $A_{h}$ be closed and convex is without loss of generality.

${ }^{14}$ The continuity of the preference relation implies the existence of such an element.
} 
Lemma 1: Given $\left(\left(A_{h}\right)_{h \in H}\right)$, where each set $A_{h}$ satisfies I.3, there exists an $\widehat{N}$ such that for all $h \in H, \quad U^{h}\left[y^{A_{h}}+\omega_{h ; 1}, z^{A_{h}}+\omega_{h ; 2}\right]>E U_{t}^{h}[s()$.$] for all t>\widehat{N}$.

Information $\mathbf{I . 4}$ "Preferences :" $1 / N \in(0,1 / \widehat{N}]$ is known.

Remark 4: We have directly imposed the condition that a number greater than $\widehat{N}$ is known. We note that one can provide informational assumptions on preferences which allow for the computation of $\widehat{N}$; however, we do not present them since doing so would call for a lot of additional notation without adding much which is conceptually novel.

\subsection{Finite Pareto optimal improvements}

We are now ready to prove the Theorem. Before doing so, we give an informal sketch of the argument.

We construct a particular Pareto improvement by (i) following the Expected allocation until some period later than $N$, then (ii) modifying the Expected allocation by transfering a net amount of at most $\delta^{*}$ from the present young to the present old within each type of agent (A.3 and Proposition 2 guarantee that we have an improvement), and finally (iii) "jumping" to a "target" stationary allocation, a point in the set $A_{h}$ for each type $h$. The informational assumptions made guarantee that the net trades of this improvement can be computed; we think of it being implemented by real lump sum taxes and transfers. Now agents are allowed to trade in competitive markets with their new endowments, i.e. post tax endowments. We show that the induced nonmonetary perfect foresight equilibria (so equilibria with loan markets only usually called the "inside money" case) are Pareto optimal since (i) the target is reached in a finite number of periods so the optimality of the allocation is essentially determined by the "tail behaviour" of the allocation, (ii) once every agent type receives the target stationary net trade as endowment, the continuation competitive allocation is necessarily Pareto optimal, and (iii) intragenerational trade gives intratemporal efficiency.

Essentially, the result calls for a variety of policy interventions in the various phases of the move to the Pareto improvement since (i) above corresponds to introducing a risk sharing arrangement across types of agents and across generations, (ii) above corresponds to an income transfer scheme within types, and (iii) above cuts the gordian knot of the infinity. Finally, trade in competitive loans markets by the different types of agents exhausts any remaining gains fron trade in each phase.

Remark 5: For "generic" economies, the set of loan market equilibria when every agent type receives his target stationary net trade as endowment, is finite. The continuation equilibrium prices are a selection from this set. Hence, if this set reduces to a singleton, we get an optimal stationary allocation in all but a finite number of periods; otherwise, we get what could be called a quasi-stationary allocation in the sense that it is a sequence with finite range. Trivially, the equilibrium is unique if either the target "Golden Rule" vector is known for each type or if the target itself is Pareto optimal, i.e. the gradients 
of the different agents utility functions point in the same direction.

Remark 6: Notice that we restrict our attention to nonmonetary equilibria (the "inside money" case). We could also consider the monetary equilibria of the model (the "outside money" case) with the redefined endowments or with the original specification of endowments. In this case, due to the well known indeterminacy problem that crops up in OLG economies (which occurs in one good economies only when there is money), we would get a plethora of equilibria. In particular, we would lose the quasi-stationarity property of the continuation equilibrium and would not be able to assert that every competitive equilibrium leads to a Pareto optimal allocation. Of course, by the Second Welfare Theorem, the allocations that we obtain in the Theorem can be supported as monetary equilibria with the original specification of endowments; but, as we noted, we cannot guarantee that a Pareto suboptimal allocation will not obtain at a monetary equilibrium. ${ }^{15}$ So we "demonetize" the economy with good reason since there is no other way of guaranteeing Pareto optimality of the improvement that we construct.

Theorem: Given assumptions A.1, A.2, and A.3, the information summarized in I.1, I.2, I.3, and I.4, allows the computation of the net trades of the Uniform allocation constructed below. The nonmonetary perfect foresight equilibria obtained when endowments are specified by the Uniform allocation, are Pareto optimal Pareto improvements over the SSE allocation.

\section{Proof:}

Step 1: We begin by specifying the Uniform allocation. The construction is based upon the Expected allocation, which was defined earlier, and is the same for the different types of agents. Hence, we carry out the construction for a generic type.

Step 1.1: We start by defining the "target" stationary net trades for the agent, $A_{h}^{1} \in$ $R^{2}$. Let $F_{h}$ be a set of restricted feasible stationary net trades specified as $F_{h}:=$ $\left\{(y, z) \in R^{2} \mid y+z=\left(\bar{c}_{h ; 1}^{*}-\omega_{h ; 1}\right)+\left(\bar{c}_{h ; 2}^{*}-\omega_{h ; 2}\right)\right\} \cap Q_{h}$. Also, let $\pi: R^{2} \rightarrow R^{1}$ be a projection function, $\pi(y, z)=y$, and for any closed set $A_{h}$ as specified in I.3, define $A_{h}^{1}:=\operatorname{argmin} \pi\left(A_{h} \cap F_{h}\right)$ so that, in geometric terms, $A_{h}^{1}$ is the north-west corner of the set $A_{h} \cap F_{h}$. Denote this net trade by $\left(y_{h}^{1}, z_{h}^{1}\right):=A_{h}^{1}$.

Step 1.2: Define the net trades of the Uniform allocation as follows:

$$
\begin{aligned}
& \widehat{z}_{h ; 0}=\bar{c}_{h ; 0}-\omega_{h ; 2}=z_{h}^{s} \text { where } s \text { is the starting state; } \\
& \left(\widehat{y}_{h ; t}, \widehat{z}_{h ; t}\right)=\left(\bar{c}_{h ; 1, t}-\omega_{h ; 1}, \bar{c}_{h ; 2, t}-\omega_{h ; 2}\right), \quad \text { for all } \quad t=1,2, \cdots, T_{h}-1
\end{aligned}
$$

where $T_{h} \geq N$ is defined by the condition

(i) $T_{h}$ is the first date $t \geq N$ at which $\bar{c}_{h ; 2, t}-\omega_{h ; 2} \leq z_{h}^{1}+\delta$, in which case the allocation is given by

\footnotetext{
${ }^{15}$ This problem is related to the well known problem about the relation between competitive monetary equilibria and Pareto optimal allocations; the problem is very difficult in all cases except when there is only one good and only one type of agent.
} 


$$
\begin{aligned}
& \left(\widehat{y}_{T_{h}}, \widehat{z}_{T_{h}}\right)=\left(\bar{c}_{h ; 1, T_{h}}-\omega_{h ; 1}, z_{h}^{1}\right), \\
& \left(\widehat{y}_{h ; t}, \widehat{z}_{h ; t}\right)=\left(y_{h}^{1}, z_{h}^{1}\right), \quad \text { for all } \quad t>T_{h} ;
\end{aligned}
$$

(ii) $T_{h}$ is such that for all $t \geq T_{h}, \bar{c}_{h ; 2, t}-\omega_{h ; 2}>z_{h}^{1}+\delta$ and $\bar{c}_{h ; 1, t}-\omega_{h ; 1}<y_{h}^{1}-\delta$, which, by the convergence result for the Expected allocation, Lemma 4.1 (ii), must occur if we are not in case (i). So $T_{h}$ is well defined for each type.

In this second case the allocation is given by

$$
\begin{aligned}
& \left(\widehat{y}_{h ; T_{h}}, \widehat{z}_{h ; T_{h}}\right)=\left(\bar{c}_{h ; 1, T_{h}}-\omega_{h ; 1}, \bar{c}_{h ; 2, T_{h}}-\omega_{h ; 2}-\delta\right), \\
& \left(\widehat{y}_{h ; t}, \widehat{z}_{h ; t}\right)=\left(\bar{c}_{h ; 1, t}-\omega_{h ; 1}+\left(t-T_{h}\right) \delta, \bar{c}_{h ; 2, t}-\omega_{h ; 2}-\left(t-T_{h}\right) \delta-\delta\right), \\
& \quad \text { for all } t=T_{h}+1, \ldots, \widehat{T}_{h}-1, \\
& \left(\widehat{y}_{h ; \widehat{T}_{h}}, \widehat{z}_{h ; \widehat{T}_{h}}\right)=\left(\bar{c}_{h ; 1, \widehat{T}_{h}}-\omega_{h ; 1}+\left(\widehat{T}_{h}-N\right) \delta, z_{h}^{1}\right), \\
& \left(\widehat{y}_{h ; t}, \widehat{z}_{h ; t}\right)=\left(y_{h}^{1}, z_{h}^{1}\right), \quad \text { for all } t>\widehat{T}_{h},
\end{aligned}
$$

where $\widehat{T}_{h}$ is such that $\bar{c}_{h ; 2, \widehat{T}_{h}}-\omega_{h ; 2}-\left(\widehat{T}_{h}+1-N\right) \delta \leq z_{h}^{1}$.

Step 2: We check that computation of the Uniform allocation is informational feasible given our hypotheses.

The information specified in I.1 (the state contingent money demands - equivalently, the SSE net trades - and the transition matrix are known), and I.3 (some sets $\left(\left(A_{h}\right)_{h \in H}\right)$ are known - this corresponds to some weak information regarding the location of the target Golden Rule) allow the computation of the target net trade vector $\left(y_{h}^{1}, z_{h}^{1}\right)$. Furthermore, Lemma 4.2 shows that $\left(y_{h}^{1}+\omega_{h ; 1}, z_{h}^{1}+\omega_{h ; 2}\right) \in R_{++}^{2}$. So, Step 1.1 is informationally feasible.

The information specified in I.1 allows the computation of the net trades of the expected allocation; that in I.1 and I.3 allows the computation of the target net trade (as indicated above); that in I.1, the (computed) target net trade, and I.2 (interval type information on $\delta^{*}$ ) and I.4 (interval type information about a preference parameter) allows the computation of $T_{h}$, in both the cases, and $\widehat{T}_{h}$ in the second case. So, Step 1.2 is informationally feasible.

The Uniform allocation that we have constructed is now given to the agents as their "new" endowment stream; informational feasibility implies that the net trades of these new endowments can be induced through real taxes.

Step 3: We verify that the Uniform allocation is a Pareto improvement over the SSE.

By Proposition 1, the Expected allocation is an improvement over the SSE allocation so that the agents of generations 1 through $T_{h}-1$ are being improved by the Uniform allocation. By Lemma 1 and Lemma 4.2, since the net trade $\left(y_{h}^{1}, z_{h}^{1}\right)$ is given to agents born after period $T_{h}$ in case (i) where $T_{h} \geq N$ (agents born after period $\widehat{T}_{h}$ in case (ii) where again $\left.\widehat{T}_{h} \geq N\right)$, all these agents are also being improved since $U^{h}\left[y_{h}^{1}+\right.$ $\left.\omega_{h ; 1}, z_{h}^{1}+\omega_{h ; 2}\right] \geq U^{h}\left[y^{A_{h}}+\omega_{h ; 1}, z^{A_{h}}+\omega_{h ; 2}\right]$. Also in case (i), from monotonicity, the agent of generation $T_{h}$ is being improved. We now show that in case (ii) the agents of generations $T_{h}$ through $\widehat{T}_{h}$ are also improved. 
We note that our assumption of normality, A.3, and Lemma 4.2 imply that

$$
\begin{gathered}
z \geq z_{h}^{1}, y \leq y_{h}^{1} \Rightarrow \frac{U_{1}^{h}\left[y+\omega_{h ; 1}, z+\omega_{2 ; h}\right]}{U_{2}^{h}\left[y+\omega_{h ; 1}, z+\omega_{h ; 2}\right]} \geq 1 \Rightarrow \\
\Rightarrow \quad U_{1}^{h}\left[y+\omega_{h ; 1}, z+\omega_{h ; 2}\right]-U_{2}^{h}\left[y+\omega_{h ; 1}, z+\omega_{h ; 2}\right] \geq 0 .
\end{gathered}
$$

This in turn implies that if $(z-n \delta) \geq z_{h}^{1}$ and $(y+n \delta) \leq y_{h}^{1}$, then $U^{h}\left[y+n \delta+\omega_{h ; 1}, z-\right.$ $\left.n \delta+\omega_{h ; 2}\right] \geq U^{h}\left[y+\omega_{h ; 1}, z+\omega_{h ; 2}\right]$ where $n$ and $\delta$ are positive numbers; also, from I.2 and Proposition 2,

$$
U^{h}\left[\bar{c}_{h ; 1, t}, \bar{c}_{h ; 2, t}-\delta\right] \geq E U_{t}^{h}[s(.)] \forall t=1,2,3, \cdots .
$$

These two facts taken together imply that if $\quad\left(\bar{c}_{h ; 2, t}-\omega_{h ; 2}-(n+1) \delta\right) \geq z_{h}^{1} \quad$ and $\quad\left(\bar{c}_{h ; 1, t}-\right.$ $\left.\omega_{h ; 1}+n \delta\right) \leq y_{h}^{1}$, then $U^{h}\left[\bar{c}_{h ; 1, t}+n \delta, \bar{c}_{h ; 2, t}-(n+1) \delta\right] \geq U^{h}\left[\bar{c}_{h ; 1, t}, \bar{c}_{h ; 2, t}-\delta\right] \geq E U_{t}^{h}[s()$. thus demonstrating that in case (ii) we have at least a weak improvement.

Step 4: We verify that any nonmonetary perfect foresight equilibrium obtained when the Uniform allocation is used as the endowment specification, is a Pareto optimal allocation.

We start by noting that beyond a certain $\bar{t}$ every agent type gets a stationary endowment specified by the vector $\left(\left(y_{h}^{1}+\omega_{h ; 1}, z_{h}^{1}+\omega_{h ; 2}\right)_{h \in H}\right)$. This follows since compactness of the feasible set implies that $\widehat{T}_{h}$ is finite (whenever we are in case (ii)), and by setting $\widehat{T}_{h}=T_{h}$ (in the case of agent types for whom we are in case (i)).

In any given period $t$ define

$Q_{t}:=\left\{q \in R_{++} \mid \sum_{h \in H} c_{h ; 1, t}(q)=\sum_{h \in H}\left(\widehat{y}_{h ; t}+\omega_{h ; 1}\right)\right.$

where $\left.\quad\left(c_{h ; 1, t}(q), c_{h ; 2, t}(q)\right) \in \operatorname{argmax}_{\left\{\left(c_{1}, c_{2}\right) \in R_{+}^{2} \mid q\left(c_{1}-\widehat{y}_{h ; t}-\omega_{h ; 1}\right)+\left(c_{2}-\widehat{z}_{h ; t}-\omega_{h ; 2}\right)=0\right\}} U^{h}\left[c_{1}, c_{2}\right]\right\}$.

It is well known that given assumptions A.1 and A.2, $Q_{t}$ is nonempty for every $t \geq 1$ though it need not be a singleton.

Let $\left\{\tilde{q}_{t}\right\}_{t \geq 1}$ be such that $\tilde{q}_{t} \in Q_{t}$ for all $t \geq 1$, i.e. a selection from the sequence of sets defined above. Now define $\tilde{p}_{1}:=\tilde{p}$ and, for $t \geq 2, \tilde{p}_{t+1}:=\tilde{p}_{t} \cdot \frac{1}{\tilde{q} t}$, inductively. If we consider the price sequence $\left\{\tilde{p}_{t}\right\}_{t \geq 1}$, we get a perfect foresight equilibrium with the additional property that

$$
\sum_{h \in H} c_{h ; 1, t}\left(\frac{\tilde{p}_{t}}{\tilde{p}_{t+1}}\right)=\sum_{h \in H}\left(\widehat{y}_{h ; t}+\omega_{h ; 1}\right) \quad \text { for all } t \geq 1
$$

which is the defining property of "inside money" equilibria.

Now notice that for $t \geq \bar{t}$, the endowment distribution does not change anymore as a consequence of which the agents' optimization problems are the same, so are their excess demand functions and $Q_{t}=Q_{\bar{t}}$ for $t \geq \bar{t}$.

Furthermore, for $t \geq \bar{t}$, the gradient vector at the post-tax endowment is given by

$$
\left.\rho_{h}:=U_{1}^{h}\left[y_{h}^{1}+\omega_{h ; 1}, z_{h}^{1}+\omega_{h ; 2}\right] / U_{2}^{h}\left[y_{h}^{1}+\omega_{h ; 1}, z_{h}^{1}+\omega_{h ; 2}\right)\right]
$$

which, by virtue of Lemma 4.2 , satisfies the condition $\rho_{h} \geq 1$ for all $h \in H$. Define $\bar{\rho}:=\max _{h \in H} \rho_{h}$ and $\underline{\rho}:=\min _{h \in H} \rho_{h}$. So $\underline{\rho} \geq 1$. Trivially, there is an excess demand if $q<\underline{\rho}$ while there is an excess supply if $q>\bar{\rho}$. By continuity of the excess demand function and the intermediate value theorem, there exists $\tilde{q} \in Q_{\bar{t}}$; furthermore, for all $\tilde{q} \in Q_{\bar{t}}$ we have $\tilde{q} \geq 1$. 
Now we check for Pareto optimality by invoking the Cass criterion (see, e.g. Geanakoplos and Polemarchakis [9]). Since we have a perfect foresight equilibrium with no restriction on trades, we have in effect a complete markets equilibrium; in addition, under our assumptions the monotonicity and uniform smoothness conditions required in the Cass criterion are satisfied. The result follows by noting that since $\tilde{q}_{t} \geq 1$ for $t \geq \bar{t}$, $\tilde{p}_{t+1} \leq \tilde{p}_{t}$ for $t \geq \bar{t}$ as a consequence of which $\sum_{t \geq \bar{t}} \frac{1}{\tilde{p}_{t}}$ diverges, which is precisely the sufficient condition given in the Cass criterion.

Q.E.D.

Remark 7: Clearly, if the objective is to reach the target stationary net trade $\left(y_{h}^{1}, z_{h}^{1}\right)$ as quickly as possible in an optimal improving manner, then it is in the government's interest to be able to compute $\delta^{*}$.

Remark 8: I.1 is a mild informational assumption; I.2 and I.4 are both interval type requirements on information and are, to that extent, relatively weak. Similarly, for I.2 we can take a sufficiently small positive number. 


\section{Proofs}

Proposition 1: If $s($.$) is an SSE allocation then it is not Pareto optimal.$

Proof: We verify that the Expected allocation, $\left\{\left(\left(\bar{c}_{h ; 1, t}, \bar{c}_{h ; 2, t}\right)_{h \in H}\right)\right\}_{t=1}^{t=+\infty}$ and $\left(\left(\bar{c}_{h ; 2,0}\right)_{h \in H}\right)$, is feasible.

$$
\begin{aligned}
& \sum_{h \in H} \bar{c}_{h ; 1, t+1}+\sum_{h \in H} \bar{c}_{h ; 2, t}=\sum_{h \in H} \pi_{t+1}^{s \alpha} c_{h ; 1}^{\alpha}+\sum_{h \in H} \pi_{t+1}^{s \beta} c_{h ; 1}^{\beta}+ \\
& \quad+\sum_{h \in H} \pi_{t}^{s \alpha}\left(\pi^{\alpha \alpha} c_{h ; 2}^{\alpha \alpha}+\pi^{\alpha \beta} c_{h ; 2}^{\alpha \beta}\right)+\sum_{h \in H} \pi_{t}^{s \beta}\left(\pi^{\beta \alpha} c_{h ; 2}^{\beta \alpha}+\pi^{\beta \beta} c_{h ; 2}^{\beta \beta}\right) \\
& =\left(\pi_{t}^{s \alpha} \pi^{\alpha \alpha}+\pi_{t}^{s \beta} \pi^{\beta \alpha}\right) \sum_{h \in H} c_{h ; 1}^{\alpha}+\left(\pi_{t}^{s \alpha} \pi^{\alpha \beta}+\pi_{t}^{s \beta} \pi^{\beta \beta}\right) \sum_{h \in H} c_{h ; 1}^{\beta}+ \\
& \quad+\pi_{t}^{s \alpha} \pi^{\alpha \alpha} \sum_{h \in H} c_{h ; 2}^{\alpha \alpha}+\pi_{t}^{s \beta} \pi^{\beta \alpha} \sum_{h \in H} c_{h ; 2}^{\beta \alpha}+\pi_{t}^{s \alpha} \pi^{\alpha \beta} \sum_{h \in H} c_{h ; 2}^{\alpha \beta}+\pi_{t}^{s \beta} \pi^{\beta \beta} \sum_{h \in H} c_{h ; 2}^{\beta \beta} \\
& =\pi_{t}^{s \alpha} \pi^{\alpha \alpha}\left(\sum_{h \in H} c_{h ; 1}^{\alpha}+\sum_{h \in H} c_{h ; 2}^{\alpha \alpha}\right)+\pi_{t}^{s \beta} \pi^{\beta \alpha}\left(\sum_{h \in H} c_{h ; 1}^{\alpha}+\sum_{h \in H} c_{h ; 2}^{\beta \alpha}\right)+ \\
& \quad+\pi_{t}^{s \alpha} \pi^{\alpha \beta}\left(\sum_{h \in H} c_{h ; 1}^{\beta}+\sum_{h \in H} c_{h ; 2}^{\alpha \beta}\right)+\pi_{t}^{s \beta} \pi^{\beta \beta}\left(\sum_{h \in H} c_{h ; 1}^{\beta}+\sum_{h \in H} c_{h ; 2}^{\beta \beta}\right) \\
& =\left(\pi_{t}^{s \alpha}\left(\pi^{\alpha \alpha}+\pi^{\alpha \beta}\right)+\pi_{t}^{s \beta}\left(\pi^{\beta \alpha}+\pi^{\beta \beta}\right)\right)\left(\sum_{h \in H} \omega_{h ; 2}+\sum_{h \in H} \omega_{h ; 2}\right) \\
& =\sum_{h \in H} \omega_{h ; 2}+\sum_{h \in H} \omega_{h ; 2} \quad s \in\{\alpha, \beta\},
\end{aligned}
$$

since market clearing implies that

$$
\sum_{h \in H} c_{h ; 1}^{s^{\prime}}+\sum_{h \in H} c_{h ; 2}^{s s^{\prime}}=\sum_{h \in H} \omega_{h ; 2}+\sum_{h \in H} \omega_{h ; 2}
$$

for $s \in\{\alpha, \beta\}$ and $s^{\prime} \in\{\alpha, \beta\}$. Hence, the Expected allocation is feasible.

By A.1 (convexity), and (ii) and (iii) in Definition 1, it is strictly improving for all agents.

Q.E.D.

Lemma 4.1: (i) $\quad \lim _{t \rightarrow+\infty} \pi_{t}^{s \alpha}:=\pi^{* s \alpha}=\pi^{\alpha} ; \lim _{t \rightarrow+\infty} \pi_{t}^{s \beta}:=\pi^{* s \beta}=\pi^{\beta}$;

(ii) $\lim _{t \rightarrow+\infty}\left(\bar{c}_{h ; 1, t}, \bar{c}_{h ; 2, t}\right):=\left(\bar{c}_{h ; 1}^{*}, \bar{c}_{h ; 2}^{*}\right)$ for all $h \in H$;

(iii) $\lim _{t \rightarrow+\infty} E U_{t}^{h}[s()]=.E^{*} U^{h}[s()$.$] for all h \in H$;

(iv) $U^{h}\left[\bar{c}_{h ; 1}^{*}, \bar{c}_{h ; 2}^{*}\right]>E^{*} U^{h}[s()$.$] for all h \in H$.

Proof: (i) Since $\Pi$ is a strictly positive stochastic matrix (by Definition 1 (iii)), 1 is it's Frobenius root. The result follows.

(ii) and (iii) Follows from the fact that the Expected allocation and the sequence of expected utilities from the SSE are linear in the conditional probabilities which converge by (i).

(iv) Both sides of the inequality are well defined by virtue of (ii) and (iii) above. Furthermore the outcome $\left(\bar{c}_{h ; 1}^{*}, \bar{c}_{h ; 2}^{*}\right)$ is the expected value of the stochastic outcome on the right hand side of the inequality; by Definition 1 (ii) it is non-degenerate. The result follows from A.1 (Jensen's Inequality).

Q.E.D.

Proposition 2: ${ }^{16}$ There exists a finite number $\delta^{*}>0$ such that, uniformly in $t$, $\forall \delta \in\left(0, \delta^{*}\right], \forall h \in H,\left(c_{h ; 1}, c_{h ; 2}\right) \in B_{\delta}\left(\bar{c}_{h ; 1, t}, \bar{c}_{h ; 2, t}\right) \quad \Rightarrow \quad U^{h}\left[c_{h ; 1}, c_{h ; 2}\right] \geq E U_{t}^{h}[s()$.$] .$

Proof: For any finite $t>0$, by Jensen's Inequality, $U^{h}\left[\bar{c}_{h ; 1, t}, \bar{c}_{h ; 2, t}\right]>E U_{t}^{h}[s()$.$] . Define$ the compact set $K$ as

$$
K:=\left\{\left(c_{1}, c_{2}\right) \in R_{+}^{2} \mid c_{1} \leq \sum_{h \in H}\left(\omega_{h ; 1}+\omega_{h ; 2}\right), \quad c_{2} \leq \sum_{h \in H}\left(\omega_{h ; 1}+\omega_{h ; 2}\right)\right\},
$$

\footnotetext{
${ }^{16}$ Notation: $B_{r}\left(x_{1}, x_{2}\right)$ denotes the closed ball of radius $r$ with center at $\left(x_{1}, x_{2}\right)$ and $\|\cdot\|$ is the usual Euclidean metric.
} 
and define $A_{h ; t}:=\left\{\left(c_{1}, c_{2}\right) \in R_{+}^{2} \mid U^{h}\left[c_{1}, c_{2}\right] \leq E U_{t}^{h}[s()].\right\} \cap K$. Now define $\delta_{h ; t}:=$ $\min _{\left(c_{1}, c_{2}\right) \in A_{h ; t}}\left\|\left(\bar{c}_{h ; 1, t}, \bar{c}_{h ; 2, t}\right)-\left(c_{1}, c_{2}\right)\right\|$. For all $h \in H, \delta_{h ; t}>0$ exists since $A_{h ; t}$ is compact.

Choose $\epsilon, \hat{\epsilon}>0$, which can depend on $h$, sufficiently small so as to construct $A_{h ; \epsilon}$ and $B_{h ; \hat{\epsilon}}\left(\bar{c}_{h ; 1}^{*}, \bar{c}_{h ; 2}^{*}\right)$, compact subsets of $R_{+}^{2}$, which are disjoint and for some large $M_{h ; \epsilon, \hat{\epsilon}}$ and for all $t>M_{h ; \epsilon, \hat{\epsilon}}\left(U^{h}\right)^{-1}\left(E U_{t}^{h}[s()].\right) \cap K \subset A_{\epsilon}^{h}$ and $\left(\bar{c}_{h ; 1, t}, \bar{c}_{h ; 2, t}\right) \in B_{h ; \hat{\epsilon}}\left(\bar{c}_{h ; 1}^{*}, \bar{c}_{h ; 2}^{*}\right)$ where $A_{h ; \epsilon}:=\left(U^{h}\right)^{-1}\left(\left[E^{*} U^{h}[s()]-.\epsilon, E^{*} U^{h}[s()]+.\epsilon\right]\right) \cap K$. That two such sets can be constructed for each type $h$, follows from Lemma 4.1 .

Now define $\widehat{\delta}_{h}:=\min _{\left(c_{1}, c_{2}\right) \in A_{h ; \epsilon}} \min _{b \in B_{h ; \hat{\epsilon}}}\left\|b-\left(c_{1}, c_{2}\right)\right\|$. The compactness of the sets and the continuity of the metric imply that $\widehat{\delta}_{h}$ is well defined; since the sets are disjoint, $\widehat{\delta}_{h}>0$. Let $\bar{\delta}_{h}:=\min \left\{\delta_{h ; 1}, \delta_{h ; 2}, \cdots, \delta_{\bar{M}_{h}}, \widehat{\delta}_{h}\right\}$ for some $\bar{M}_{h}>M_{h ; \epsilon, \hat{\epsilon}}$; hence $\bar{\delta}_{h}>0$. Now consider the set $\left[\bar{\delta}_{h}, \delta_{h ; 1}\right]$ and let $\bar{\delta}_{h ; T}:=\min \left\{\delta_{h ; 1}, \ldots, \delta_{h ; T}\right\}$. Clearly, $\bar{\delta}_{h ; T} \in\left[\bar{\delta}_{h}, \delta_{h ; 1}\right]$ for all $T$ and the sequence $\left\{\bar{\delta}_{h ; T}\right\}_{T=1}^{T=+\infty}$ is weakly monotone decreasing so it has a limit. Finally, define $\delta_{h}^{*}$ to be $\delta_{h}^{*}:=\lim _{T \rightarrow \infty} \bar{\delta}_{h ; T}$ and set $\delta^{*}:=\min _{h \in H} \delta_{h}^{*}$.

Q.E.D.

Lemma 1: Given $\left(\left(A_{h}\right)_{h \in H}\right)$, where each set $A_{h}$ satisfies I.3, there exists an $\widehat{N}$ such that for all $h \in H, \quad U^{h}\left[y^{A_{h}}+\omega_{h ; 1}, z^{A_{h}}+\omega_{h ; 2}\right]>E U_{t}^{h}[s()$.$] for all t>\widehat{N}$.

Proof: By I.3 (ii) and (iii), and the definition of $\left(y^{A_{h}}, z^{A_{h}}\right), U\left[y^{A_{h}}+\omega_{h ; 1}, z^{A_{h}}+\omega_{h ; 2}\right]>$ $E^{*} U^{h}[s()$.$] . So Lemma 4.1$ (iii) and the continuity of $E U_{t}^{h}[s()$.$] in \pi_{t}^{s \alpha}$ and $\pi_{t}^{s \beta}$ imply that for each type $h \in H$, there exists an $N^{A_{h}}$ such that $U^{h}\left[y^{A_{h}}+\omega_{h ; 1}, z^{A_{h}}+\omega_{h ; 2}\right]>$ $E U_{t}^{h}[s()$.$] for all t>N^{A_{h}}$. The result follows by defining $\widehat{N}:=\max _{h \in H} N^{A_{h}}$. Q.E.D.

Lemma 4.2: For all $\left.h \in H, U_{1}^{h}\left[y_{h}^{1}+\omega_{h ; 1}, z_{h}^{1}+\omega_{h ; 2}\right] / U_{2}^{h}\left[y_{h}^{1}+\omega_{h ; 1}, z_{h}^{1}+\omega_{h ; 2}\right)\right]=\rho_{h} \geq 1$. Proof: It is easily checked that $A_{h} \cap F_{h}$ is compact, so that $\left(y_{h}^{1}, z_{h}^{1}\right)$ is well defined. We now show that $\left(y_{h}^{1}+\omega_{h ; 1}, z_{h}^{1}+\omega_{h ; 2}\right) \in R_{++}^{2}$ so that by A.1 the MRS at $\left(y_{h}^{1}, z_{h}^{1}\right)$ is well defined. By our boundary assumption on preferences, all the sunspot outcomes are in $R_{++}^{2}$ implying that $A_{h}^{*}+\left\{\left(\omega_{h ; 1}, \omega_{h ; 2}\right)\right\}$ stays away from the boundary of $R_{+}^{2}$ so that $A_{h}^{*} \cap F_{h} \neq \emptyset$. By I.3, $\left(\left(c_{h ; 1}^{G R}\left(\bar{c}_{h ; 1}^{*}+\bar{c}_{h ; 2}^{*}\right)-\omega_{h ; 1}, c_{h ; 2}^{G R}\left(\bar{c}_{h ; 1}^{*}+\bar{c}_{h ; 2}^{*}\right)-\omega_{h ; 2}\right) \in A_{h} \cap F_{h}\right.$ and $A \cap A^{*}=\emptyset$, implying that $\left(y_{h}^{1}+\omega_{h ; 1}, z_{h}^{1}+\omega_{h ; 2}\right) \in R_{++}^{2}$. Now note that by A.1 (convexity and monotonicity) $\left.U_{1}^{h}\left[y_{h}^{1}+\omega_{h ; 1}, z_{h}^{1}+\omega_{h ; 2}\right] / U_{2}^{h}\left[y_{h}^{1}+\omega_{h ; 1}, z_{h}^{1}+\omega_{h ; 2}\right)\right]=\rho_{h} \geq$ $1=U_{1}^{h}\left[\left(\left(c_{h ; 1}^{G R}\left(\bar{c}_{h ; 1}^{*}+\bar{c}_{h ; 2}^{*}\right), c_{h ; 2}^{G R}\left(\bar{c}_{h ; 1}^{*}+\bar{c}_{h ; 2}^{*}\right)\right)\right] / U_{2}\left[\left(\left(c_{h ; 1}^{G R}\left(\bar{c}_{h ; 1}^{*}+\bar{c}_{h ; 2}^{*}\right), c_{h ; 2}^{G R}\left(\bar{c}_{h ; 1}^{*}+\bar{c}_{h ; 2}^{*}\right)\right)\right]\right.\right.$ since $\left(y_{h}^{1}, z_{h}^{1}\right)=\operatorname{argmin} \pi\left(A_{h} \cap F_{h}\right)$ and $\left(\left(c_{h ; 1}^{G R}\left(\bar{c}_{h ; 1}^{*}+\bar{c}_{h ; 2}^{*}\right)-\omega_{h ; 1}, c_{h ; 2}^{G R}\left(\bar{c}_{h ; 1}^{*}+\bar{c}_{h ; 2}^{*}\right)-\omega_{h ; 2}\right) \in A_{h} \cap F_{h}\right.$.

Q.E.D. 


\section{References}

[1] Azariadis, C.: Self-Fulfilling Prophecies. Journal of Economic Theory 25, 380-396 (1981)

[2] Cass, D., Shell, K.: Do Sunspots Matter? Journal of Political Economy 91, 189-227

[3] Chattopadhyay, S. K.: Pareto Optimal Improvements for Sunspots: The Golden Rule as a Target for Stabilization. Economic Theory 8, 122-135 (1996)

[4] Chattopadhyay, S. K.: Information, Stabilization and Welfare: the Case of Sunspots. Working Paper No. 281, Dept. of Economics, The Hebrew University of Jerusalem 1993 (and mimeo. University of Alicante 1997)

[5] Chiappori, P. A., Guesnerie, R.: Sunspot Equilibria in Market Models. In: Hildenbrand, W., Sonnenschein, H. (eds.) Handbook of Mathematical Economics, IV. New York: North Holland 1991

[6] Geanakoplos, J. D.: Overlapping Generations Models of General Equilibrium. In: Eatwell, J., Milgate, M., Newman, P. (eds.) The New Palgrave Dictionary of Economics: General Equilibrium. London: MacMillan 1989

[7] Geanakoplos, J. D., Polemarchakis, H. M.: Existence, Regularity and Constrained Suboptimality of Competitive Allocations When Markets are Incomplete. In Heller, W., Starett, D., Starr, R. (eds.) Uncertainty, Information and Communication: Essays in Honour of K. J. Arrow, 3. Cambridge: Cambridge University Press (1986)

[8] Geanakoplos, J. D., Polemarchakis, H. M.: Observability and Optimality. Journal of Mathematical Economics, 19, 153-165 (1990)

[9] Geanakoplos, J. D., Polemarchakis, H. M.: Overlapping Generations. In: Hildenbrand, W., Sonnenschein, H. (eds.) Handbook of Mathematical Economics, IV. New York: North Holland 1991

[10] Grandmont, J. M.: On Endogenous Competitive Business Cycles. Econometrica, 53, 995-1045 (1985)

[11] Grandmont, J. M.: Stabilizing Competitive Business Cycles. Journal of Economic Theory, 40, 57-76 (1986)

[12] Guesnerie, R., Woodford, M.: Endogenous Fluctuations. In Laffont, J. J. (ed) Advances in Economic Theory, II. Cambridge: Cambridge University Press (1992)

[13] Peck, J.: On The Existence of Sunspot Equilibria in an Overlapping Generations Model. Journal of Economic Theory, 44, 19-42 (1988)

[14] Woodford, M.: Stationary Sunspot Equilibria in a Finance Constrained Economy. Journal of Economic Theory, 40, 128-137 (1986) 